\title{
$\alpha 2 \delta-2$ is Required for Depolarization-induced Suppression of Excitation in Purkinje cells
}

Kathleen A. Beeson ${ }^{1,2}$, Gary L. Westbrook ${ }^{3}$, Eric Schnell ${ }^{2,4^{*}}$

1 Neuroscience Graduate Program, OHSU, Portland, OR, 97239; 2 Department of

Anesthesiology and Perioperative Medicine, OHSU, Portland, OR, 97239; 3 Vollum Institute, OHSU, Portland, OR, 97239; 4 Operative Care Division, Portland VA Health Care System,

Portland, OR, 97239

* Eric Schnell, MD, PhD

Email: schneler@ohsu.edu

Author Contributions: KAB designed and performed research, analyzed data and wrote the original paper; GLW reviewed and edited paper; ES designed research, reviewed and edited paper.

Competing Interest Statement: The authors declare no competing financial interests.

Classification: Biological Sciences; Neuroscience.

Keywords: $\alpha 2 \delta-2$ proteins, calcium channels, depolarization-induced suppression of excitation, nanodomains, Purkinje cells. 


\begin{abstract}
$\alpha 2 \delta$ proteins (CACNA2D1-4) are required for normal neurological function, although how they control neuronal output remains unclear. Using whole-cell recordings of mouse Purkinje cells, we show $\alpha 2 \delta-2$ is required for functional coupling of postsynaptic voltage-dependent calcium entry with effector mechanisms controlling two different outputs, depolarization-induced suppression of excitation mediated by endocannabinoid signaling, and spike afterhyperpolarization generated by calcium-dependent potassium channels. Our findings indicate an important role for $\alpha 2 \delta-2$ proteins in regulating functional postsynaptic calcium channel-coupling in neurons.
\end{abstract}

\title{
Significance Statement
}

Calcium influx via membrane voltage-dependent calcium channels drives numerous neuronal processes by signaling through calcium-dependent effector molecules. Signal precision is achieved in part by calcium channel-effector coupling. In mouse Purkinje cell neurons, we show that neuronal $\alpha 2 \delta-2$ protein functionally couples calcium entry to two different postsynaptic calcium-dependent signals, retrograde endocannabinoid signaling and the action potential afterhyperpolarization. Our findings provide new insights about the control of calcium channeleffector coupling as well as new roles for $\alpha 2 \delta-2$ proteins in neurons.

\section{Main Text}

\section{Introduction}

Many intracellular signaling cascades are triggered by a common messenger: calcium entering via voltage-gated $\mathrm{Ca}^{2+}$ channels (VGCCs) on the plasma membrane. Specificity of $\mathrm{Ca}^{2+}-$ dependent signaling depends on proximity of VGCCs to effectors in functional nanodomains, and this coupling is critical for neuronal function. For example, in Purkinje cells (PCs), depolarizationinduced suppression of excitation (DSE) is initiated via $\mathrm{Ca}^{2+}$-dependent endocannabinoid release $(1,2)$. Likewise, VGCC-K $\mathrm{K}_{\mathrm{Ca}}$ coupling generates spike afterhyperpolarization (AHP), ultimately setting firing frequency $(3,4)$. Thus, molecules coupling VGCCs to effector-specific signaling critically contribute to transduction of neuronal outputs.

Auxiliary VGCC $\alpha 2 \delta$ proteins (CACNA2D1-4) contribute to VGCC membrane trafficking (5), and may couple presynaptic VGCCs to vesicle release machinery (6). However, little is known about auxiliary postsynaptic functions of $\alpha 2 \delta$ outside of these contexts, apart from developmental $\mathrm{Ca}^{2+}$-independent roles $(7,8)$. Here we report that in PCs, which exclusively express $\alpha 2 \delta-2(9,10)$, loss of $\alpha 2 \delta-2$ (CACNA2D2) disrupts two disparate forms of postsynaptic VGCC signaling, demonstrating previously unappreciated roles for $\alpha 2 \delta$ proteins in VGCC-effector coupling.

\section{Results}

At PC climbing fiber synapses, postsynaptic VGCC-mediated $\mathrm{Ca}^{2+}$ entry initiates retrograde endocannabinoid signaling, acutely reducing presynaptic release probability - a form of plasticity known as DSE $(1,2)$. Specificity of DSE signaling is achieved by tight functional coupling of postsynaptic VGCCs with $\mathrm{Ca}^{2+}$-sensitive endocannabinoid effector molecules (2). Using wholecell recordings of PCs in acutely prepared brain slices from CACNA2D2 KO and WT littermate mice, we investigated whether absence of $\alpha 2 \delta-2$ affects DSE.

In WT PCs, DSE elicited with a $250 \mathrm{~ms}$ depolarizing step reduced the amplitude of regularly evoked climbing fiber excitatory postsynaptic currents (EPSCs) by $25 \%$ (Fig 1A-E). In contrast, DSE was absent in KO PCs (Fig 1B-E). The ducky mouse, which also lacks $\alpha 2 \delta-2$ protein, has a reported $\sim 30 \%$ decrease in PC somatic VGCC current density, which is thought to represent decreased VGCC surface trafficking (11). As DSE magnitude is related to the amount of $\mathrm{Ca}^{2+}$ influx, it is possible that the reduced VGCC density prevented DSE. However, although 
increasing depolarizing step length enhances $\mathrm{Ca}^{2+}$ influx and DSE in WT mice (1), a four-fold increase in step duration still failed to evoke DSE in $\alpha 2 \delta-2$ KO PCs (Fig 1D-F).

As DSE was not rescued by increased activation of VGCCs in KO PCs, we hypothesized that functional coupling of $\mathrm{Ca}^{2+}$ influx to effector molecules was disrupted in the absence of $\alpha 2 \delta-2$. To examine this, we lowered [EGTA] in our internal solution. As expected for WT PCs, decreasing [EGTA] from $10 \mathrm{mM}$ to either $2 \mathrm{mM}$ or $0.2 \mathrm{mM}$ resulted in more profound DSE magnitude, which further increased with longer voltage steps (Fig 2A-C), consistent with greater diffusion of $\mathrm{Ca}^{2+}$ from its point of entry. Notably, reduced $\mathrm{Ca}^{2+}$ buffering restored DSE in KO PCs (Fig 2D-F), indicating that signaling mechanisms involved in DSE expression remained intact. Thus, these data indicate that rather than affecting $\mathrm{Ca}^{2+}$ entry per se, $\alpha 2 \delta-2$ mediates tight functional coupling between VGCCs and endocannabinoid release.

To resolve whether $\alpha 2 \delta-2$ affects coupling of other molecules to postsynaptic VGCC nanodomains, we focused on $\mathrm{Ca}^{2+}$-dependent action potential AHPs. In PCs, the AHP is mediated by BK-type $\mathrm{K}_{\mathrm{Ca}}$ channels $(12,13)$, and regulates $\mathrm{PC}$ firing rate $(3,4)$. To assess VGCC$\mathrm{K}_{\mathrm{Ca}}$ coupling, we recorded spontaneous spiking in PCs from WT and KO mice during whole-cell current-clamp recordings. In agreement with previous studies in ducky mutants $(11,14)$, tonic spike rate in $\alpha 2 \delta-2$ KO PCs was reduced compared to WT (Fig 3A-B). Furthermore, AHP amplitude of individual spike waveforms was consistently smaller in $\alpha 2 \delta-2 \mathrm{KO}$ cells (Fig 3C-D), indicating reduced $\mathrm{K}_{\mathrm{Ca}}$ channel activation $(4,12)$. Other membrane properties were unchanged in KO PCs, including the resting membrane potential, membrane polarization rate, spike threshold and spike height (Fig 3E-J), indicating that changes were limited to the $\mathrm{K}_{\mathrm{Ca}}$-mediated AHP. Additionally, there was no change in BK membrane localization in PCs by immunohistochemistry (Fig 3K; Membrane BK density (puncta/ $\mu \mathrm{m}$ ): WT $=0.82 \pm 0.13, \mathrm{n}=4 ; \mathrm{KO}=0.75 \pm 0.08, \mathrm{n}=3$; $\mathrm{p}$ $=0.7$; Student's unpaired t-test), consistent with normal BK expression and function, as previously described in ducky mice (15).

To determine whether the reduced AHP resulted from functionally uncoupled VGCC- KCa channels in KO mice, we dialyzed PCs with an increased EGTA concentration (from $0.5 \mathrm{mM}$ to 5 $\mathrm{mM}$ ) to uncouple VGCC-BK signaling (16). This additional $\mathrm{Ca}^{2+}$ buffering reduced the AHP amplitude in WT PCs to the same value as the KO, with no effect on KO AHP (Fig 3C-D). Thus, increased $\mathrm{Ca}^{2+}$ buffering uncoupled VGCC-K $\mathrm{K}_{\mathrm{Ca}}$ signaling in WT, but $\mathrm{K}_{\mathrm{Ca}}$ channels were already functionally uncoupled in the $\alpha 2 \delta-2 \mathrm{KO}$.

\section{Discussion}

As a primary signal in neurons, precise spatiotemporal regulation of $\mathrm{Ca}^{2+}$ influx maintains fidelity of $\mathrm{Ca}^{2+}$-dependent processes in neurons. Consequently, molecules controlling VGCC coupling to downstream effectors are critical to neuronal function. $\alpha 2 \delta$ proteins are involved trafficking of VGCCs to the plasma membrane (5). However, this conclusion was necessarily based on heterologous expression systems, as most neurons express more than one $\alpha 2 \delta$ isoform. Thus, information of postsynaptic auxiliary functional roles of $\alpha 2 \delta$ proteins in neurons is limited. Because PCs selectively express the $\alpha 2 \delta$ - 2 isoform $(9,10)$, the CACNA2D2 KO mouse provides an ideal model to examine the functional roles for $\alpha 2 \delta$ proteins in the postsynaptic compartment. Our results demonstrate that two distinct $\mathrm{Ca}^{2+}$-dependent mechanisms, DSE and $\mathrm{K}_{\mathrm{ca}}$ signaling, are disrupted in $\alpha 2 \delta-2 \mathrm{KO} P C s$, indicating functional loss of postsynaptic VGCC-effector coupling $(2,16)$.

How does $\alpha 2 \delta-2$, a largely extracellular protein, mediate functional coupling of VGCCs with intracellular effector proteins? It is possible that $\alpha 2 \delta-2$ directly associates with other extracellular proteins involved in VGCC domains (5). As endocannabinoid machinery resides at synapses (17), and $\alpha 2 \delta$ proteins are important for synapse formation $(7,9,10,18), \alpha 2 \delta$ could potentially regulate VGCC nanodomains by binding to presynaptic adhesion proteins. Another possibility is that $\alpha 2 \delta-2$ localizes VGCCs to lipid rafts. Though VGCCs are abundant in non-lipid raft membrane fractions where they are independent of $\alpha 2 \delta-2$, VGCCs and $\alpha 2 \delta-2$ colocalize in lipid rafts isolated from cerebellar homogenates (19). Intriguingly, DAG lipase- $\alpha$, the enzyme responsible for synthesis of endocannabinoids involved in DSE, has also been isolated in lipid 
rafts (17), and mislocalization of VGCCs away from lipid rafts might explain the reduced efficacy of VGCC-signaling in $\alpha 2 \delta-2 \mathrm{KO}$ PCs. The deficits in $\mathrm{Ca}^{2+}$-dependent signaling we observed may contribute to the dramatic neurologic phenotypes in $\alpha 2 \delta-2 \mathrm{KO}$ mice. Our results provide clues for future work to directly assay $\alpha 2 \delta-2$ interacting proteins and subcellular localization of VGCCs in PCs. Future investigations of the coupling roles of other $\alpha 2 \delta$ isoforms in neurons will provide valuable insights into how these proteins impact neurological functions across the brain.

\section{Materials and Methods}

Animals: Cacna2d2 knockout mice (Cacna2d2tm1Svi MGI = 3055290; generously supplied by Drs. Sergey Ivanov and Lino Tessarollo) were obtained as cryopreserved sperm and re-derived via in vitro fertilization on a C57BL/6J background. Breeding mice were kept heterozygous, and genotyping was performed as previously described (10). Mice were maintained in facilities fully accredited by the Association for Assessment and Accreditation of Laboratory Animal Care and veterinary care was provided by Oregon Health \& Science University's Department of Comparative Medicine. All animal care and experiments were performed in accordance with state and federal guidelines, and all protocols were approved by the OHSU Institutional Animal Care and Use Committee.

Slice Preparation and Electrophysiology: Male and female mice were used between the ages of p21-30. KO and WT littermates were deeply anesthetized and transcardially perfused with icecold choline-based solution containing (mM): 125 choline- $\mathrm{Cl}, 2.5 \mathrm{KCl}, 1.25 \mathrm{NaH}_{2} \mathrm{PO}_{4}, 0.44$ ascorbate, $2 \mathrm{Na}$ pyruvate, 3 3-myo-inositol, $10 \mathrm{D}$-glucose, $25 \mathrm{NaHCO}_{3}, 7 \mathrm{MgCl}_{2}, 0.5 \mathrm{CaCl}_{2}$ (osmolarity adjusted to $305 \mathrm{mOsm}$ ) and equilibrated with $95 \% \mathrm{O}_{2}$ and $5 \% \mathrm{CO}_{2}$ gas mixture. Acute $300 \mu \mathrm{m}$ sagittal slices were cut from cerebellum using a vibratome (VT1200, Leica

Microsystems), and incubated for 30 minutes in standard artificial cerebral spinal fluid (aCSF) at $34^{\circ} \mathrm{C}$.

Voltage clamp recordings: Whole-cell recordings were obtained using 1-3 $M \Omega$ borosilicate glass pipettes filled with internal solution containing (in $\mathrm{mM}$ ): $100 \mathrm{CsMeSO}_{4}, 35 \mathrm{CsCl}$, 15 TEA-Cl, $1 \mathrm{MgCl}_{2}$, 15 HEPES, 2 ATP-Mg, 0.3 TrisGTP, 10 phosphocreatine, and 2 QX-314. A large batch of this internal base solution was equally divided and 10, 2 or $0.2 \mathrm{mM}$ EGTA was added to each third. All internals were adjusted to $\mathrm{pH} 7.3$ with $\mathrm{CsOH}$ and osmolarity to 293 mOsm. External solution contained (in mM): $125 \mathrm{NaCl}, 25 \mathrm{NaHCO}_{3}, 1.25 \mathrm{NaH}_{2} \mathrm{PO}_{4}, 3 \mathrm{KCl}, 25$ Dextrose, $2 \mathrm{CaCl}_{2}, 1 \mathrm{MgCl}_{2}$ (osmolarity adjusted to $300 \mathrm{mOsm}$ ) and was continuously perfused via roller pump.

PCs were identified and recorded as previously described (10). Briefly, PCs were chosen from the vermis lobe VI, were identified by soma size and location in the PC layer, and whole-cell patch-clamp recordings were obtained in voltage clamp mode. Cell capacitance, series resistance and input resistance monitored in real time using intermittent $-10 \mathrm{mV}$ voltage steps. Inhibition was blocked in all experiments by $10 \mu \mathrm{M}$ SR95531 (Tocris), and 0.2-0.5 $\mu \mathrm{M}$ NBQX (Tocris) was included to maintain voltage clamp of climbing fiber-mediated excitatory postsynaptic currents (EPSCs). All voltage clamp recordings were performed at room temperature. Signals were amplified with a MultiClamp 700B (Molecular Devices) amplifier and pipette capacitance was compensated using MultiClamp software. Signals were low-pass filtered at $6 \mathrm{kHz}$ and sampled at $10 \mathrm{kHz}$, and digitized with a National Instruments analog-to-digital board. All recordings were acquired and analyzed using IgorPro-based (Wavemetrics) software.

For DSE experiments, PCs were held at $-70 \mathrm{mV}$ while climbing fiber-mediated EPSCs were evoked using a monopolar glass electrode in the granule cell layer. After obtaining 2 minutes of baseline responses at $0.2 \mathrm{~Hz}$, a depolarizing voltage step to $0 \mathrm{mV}$ of $1 \mathrm{~s}, 500 \mathrm{~ms}$ or $250 \mathrm{~ms}$ duration was delivered to induce DSE, after which PCs were returned to $-70 \mathrm{mV}$ and 0.2 $\mathrm{Hz}$ stimulation was continued. DSE plasticity is acute, and most synapses recover back to baseline EPSC amplitudes within $<60$ seconds (1). As a small amount of "run down" was routinely observed in the evoked CF-mediated EPSC amplitude, DSE inclusion criteria required EPSC amplitudes to return to $80 \%$ baseline within 2 minutes post-stimulation (opposed to "stepping" to decreased amplitude without recovery). A minimum of 5 minutes were waited between DSE inductions, and step length was randomized throughout experiment. Series 
resistance was not compensated; cells with series resistance $>10 \mathrm{M} \Omega$, or a $>2 \mathrm{M} \Omega$ change in series resistance over the course of the experiment were excluded.

For analysis, EPSC amplitudes were binned every 10 seconds (2 traces) and normalized to the 1 minute of baseline immediately preceding the depolarizing step. The 'DSE magnitude' (e.g. Figure 2E) is based on the average of EPSC amplitude 5 and $10 \mathrm{~s}$ after the depolarizing step. Example traces shown are from $5 \mathrm{~s}$ after the depolarizing step. A minimum of 3 mice per genotype were used for each manipulation, with no more than 2 cells/treatment coming from one mouse. For data presentation, EPSC traces were off-line box-filtered at $1 \mathrm{kHz}$ in lgor64 software.

Current clamp recordings: For spontaneous spike experiments, internal solution contained (in mM): $120 \mathrm{KCH}_{3} \mathrm{SO}_{3}, 10 \mathrm{HEPES}, 10 \mathrm{NaCl}, 2 \mathrm{MgCl}_{2}, 0.5 \mathrm{EGTA}, 4$ ATP-Mg, 0.3 TrisGTP, and 14 phosphocreatine, pH 7.35 adjusted with $\mathrm{KOH}$ (osmolarity adjusted to $293 \mathrm{mOsm}$ ). A stock of EGTA solution was added to aliquots of internal, to increase [EGTA] to $5 \mathrm{mM}$ as needed. Synaptic inhibition was achieved with $10 \mu \mathrm{M}$ SR95531 (Tocris) and $10 \mu \mathrm{M}$ NBQX (Tocris), and recordings were made at $36^{\circ} \mathrm{C}$ using an in-line heater. PCs in whole-cell mode from vermis lobe VI were first held in voltage clamp mode to monitor access series and input resistance before switching to current clamp. Changes in access were corrected with bridge balance using Multiclamp software. For increased action potential waveform resolution, some current clamp experiments were sampled at $50 \mathrm{kHz}$.

Spontaneous spikes from tonically firing PCs with $<400$ pA holding current and $<10$ MOhm series were analyzed using the Igor64 Neuromatic tools. Firing frequency data was collected from 10 seconds of recording, which yielded $\sim 100-500$ spikes. Action potential properties were assessed by averaging 50 consecutive spikes. Afterhyperpolarization (AHP) amplitude was measured as the difference between the threshold voltage $\left(\mathrm{V}_{\text {thres }}=\right.$ depolarization rate $>10 \mathrm{~V} / \mathrm{s}$ ) and the minimum voltage reached within $5 \mathrm{~ms}$ of spiking. All current clamp data was taken at least 3 minutes after break-in to allow time for internal solution to dialyze. Spike traces were box filtered for data visualization, and phase plane plots were made using lgor64.

Immunohistochemistry: Immunohistochemistry was performed as described (10). Briefly, p21 WT and KO mice were deeply anesthetized and transcardially perfused with ice-cold PBS followed by $4 \%$ paraformaldehyde (PFA)-PBS. Following decapitation, brains were removed and fixed overnight in $4 \%$ PFA-PBS, and stored in PBS at $4^{\circ} \mathrm{C}$. Sagittal cerebellar slices were made at 50 $\mu \mathrm{m}$ thickness using a vibratome, and slices containing vermis lobe $\mathrm{VI}$ were permeabilized for $1 \mathrm{hr}$ with $0.4 \%$ Triton-PBS with $10 \%$ normal horse serum at RT. Slices were stained with goat antiParvalbumin (Swant \#PVG-213; 1:1000) and mouse anti-BK (Neuromab \#73-022; 1:500) overnight at $4^{\circ} \mathrm{C}$. Corresponding fluorescently labeled secondaries (Invitrogen; 1:500) were applied after rinsing $3 x$ in PBS, and slices were mounted on glass cover slips using Fluoromount G (Sigma-Aldrich).

BK membrane expression was imaged using $63 x$ oil immersion lens on a LSM980 microscope with ZEN software. $\sim 7 \mu \mathrm{m}$ z-stack images of primary PC dendrites were acquired at $0.15 \mu \mathrm{m}$ intervals using the PV channel at $4.5 \times$ zoom with $680 \times 680$ pixel resolution. Airyscan images were processed using default settings in ZEN. Quantification of membrane localized BK puncta was done by a separate researcher, blinded to genotype, using the most transverse section of dendrite from each z-stack. For presentation, images were processed in Fij//lmageJ, illustrating $0.45 \mu \mathrm{m}$ maximum projections, and the panel was assembled using Adobe Photoshop.

Statistics: The data were tested for normality using Shapiro-Wilk test. Data from male and female mice were grouped. The difference in magnitude of DSE between the WT 250 ms depolarization step condition and other groups were compared using a one-way ANOVA with Dunnett's correction for multiple comparisons. For current clamp data, student's unpaired t-tests were used for spike frequency comparison between WT and KO. In current clamp experiments using $5 \mathrm{mM}$ EGTA, only AHP amplitude was significantly different (all other measures not shown). For this data, a one-way ANOVA with Fisher's LSD was used to compare all groups to WT $0.5 \mathrm{mM}$ condition. All electrophysiology experiments utilized at least 3 animals per genotype, where $n=\#$ cells. For immunohistochemistry of BK membrane density measurements, 2-4 images per animal were averaged $(n=$ mice), and an unpaired t-test was used for comparison. Data were graphed in Prism GraphPad version 8 and are reported as the mean \pm SEM. ${ }^{*} p<0.05,{ }^{* *} p<0.01,{ }^{* * *} p<0.001$. 


\section{Acknowledgments}

This research was supported by VA I01-BX004938 (ES), DoD W81XWH-18-1-0598 (ES), NIH T32NS007466 (KAB), NIH R01-NS080979 (GLW), NIH P30NS061800 (OHSU), and NINDS $1 R 21 N S 102948$ (Ines Koerner/ES). The contents of this manuscript do not represent the views of the U.S. Department of Veterans Affairs or the United States Government.

\section{References}

1. Brenowitz SD \& Regehr WG (2003) Calcium dependence of retrograde inhibition by endocannabinoids at synapses onto Purkinje cells. The Journal of Neuroscience 23(15):6373-6384.

2. Brenowitz SD, Best AR, \& Regehr WG (2006) Sustained elevation of dendritic calcium evokes widespread endocannabinoid release and suppression of synapses onto cerebellar Purkinje cells. J Neurosci 26(25):6841-6850.

3. Raman IM \& Bean BP (1999) lonic Currents Underlying Spontaneous Action Potentials in Isolated Cerebellar Purkinje Neurons. Journal of Neuroscience 19(5):12.

4. Womack MD, Chevez C, \& Khodakhah K (2004) Calcium-activated potassium channels are selectively coupled to P/Q-type calcium channels in cerebellar Purkinje neurons. J Neurosci 24(40):8818-8822.

5. Dolphin AC \& Lee A (2020) Presynaptic calcium channels: specialized control of synaptic neurotransmitter release. Nat Rev Neurosci 21(4):213-229.

6. Hoppa MB, Lana B, Margas W, Dolphin AC, \& Ryan TA (2012) alpha2delta expression sets presynaptic calcium channel abundance and release probability. Nature 486(7401):122-125.

7. Eroglu C, et al. (2009) Gabapentin receptor alpha2delta-1 is a neuronal thrombospondin receptor responsible for excitatory CNS synaptogenesis. Cell 139(2):380-392.

8. Kurshan PT, Oztan A, \& Schwarz TL (2009) Presynaptic alpha2delta-3 is required for synaptic morphogenesis independent of its Ca2+-channel functions. Nat Neurosci 12(11):1415-1423.

9. Lein ES, et al. (2007) Genome-wide atlas of gene expression in the adult mouse brain. Nature 445(7124):168-176.

10. Beeson KA, Beeson R, Westbrook GL, \& Schnell E (2020) alpha2delta-2 Protein Controls Structure and Function at the Cerebellar Climbing Fiber Synapse. J Neurosci 40(12):24032415.

11. Donato $\mathrm{R}$, et al. (2006) The ducky(2J) mutation in Cacna2d2 results in reduced spontaneous Purkinje cell activity and altered gene expression. J Neurosci 26(48):1257612586.

12. Edgerton JR \& Reinhart PH (2003) Distinct contributions of small and large conductance Ca2+-activated K+ channels to rat Purkinje neuron function. J Physiol 548(Pt 1):53-69.

13. Niday Z \& Bean BP (2021) BK channel regulation of after-potentials and burst firing in cerebellar Purkinje neurons. $J$ Neurosci.

14. Walter JT, Alvina K, Womack MD, Chevez C, \& Khodakhah K (2006) Decreases in the precision of Purkinje cell pacemaking cause cerebellar dysfunction and ataxia. Nat Neurosci 9(3):389-397.

15. Fell B, et al. (2016) alpha2delta2 Controls the Function and Trans-Synaptic Coupling of Cav1.3 Channels in Mouse Inner Hair Cells and Is Essential for Normal Hearing. J Neurosci 36(43):11024-11036. 
16. Fakler B \& Adelman JP (2008) Control of $\mathrm{K}(\mathrm{Ca})$ channels by calcium nano/microdomains. Neuron 59(6):873-881.

17. Rimmerman N, et al. (2008) Compartmentalization of endocannabinoids into lipid rafts in a dorsal root ganglion cell line. Br J Pharmacol 153(2):380-389.

18. Cole RL, et al. (2005) Differential distribution of voltage-gated calcium channel alpha-2 delta (alpha2delta) subunit mRNA-containing cells in the rat central nervous system and the dorsal root ganglia. J Comp Neurol 491(3):246-269.

19. Davies A, et al. (2006) The calcium channel alpha2delta-2 subunit partitions with CaV2.1 into lipid rafts in cerebellum: implications for localization and function. $J$ Neurosci 26(34):8748-8757. 
Figures and Tables
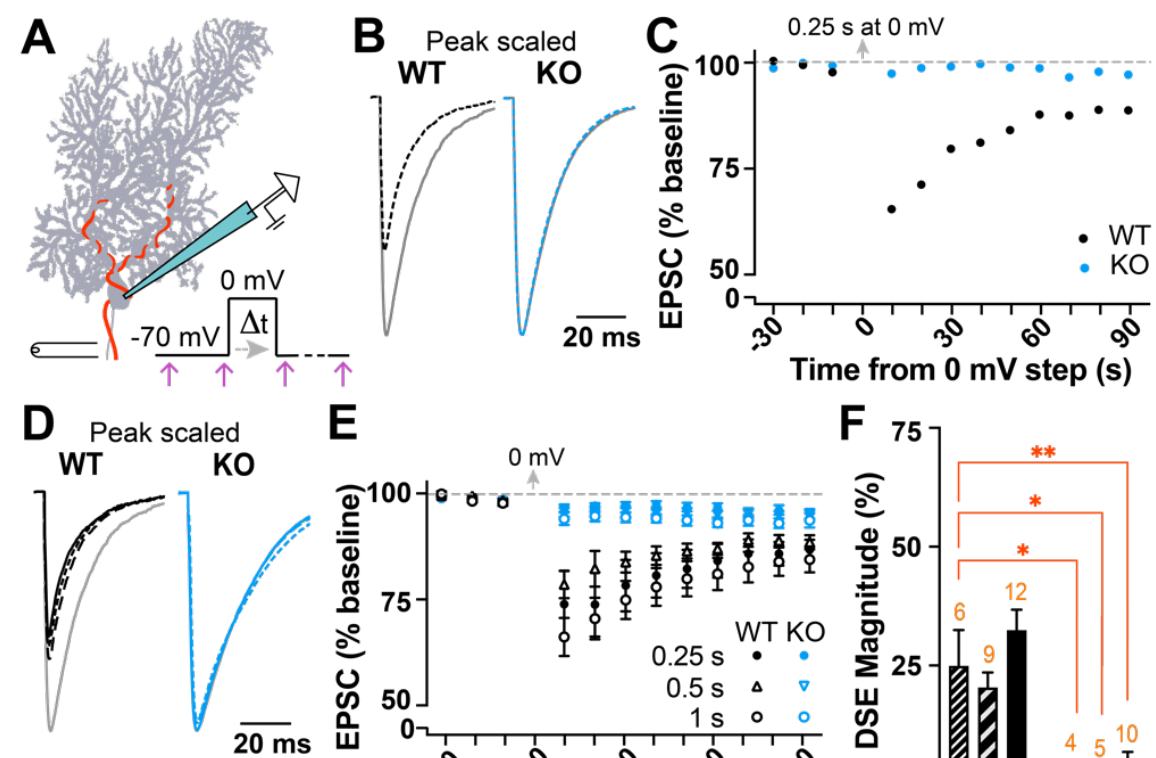

Step: $0 . \overline{2} 5 \mathrm{~s} \quad \overline{0} . \overline{\mathrm{s}} \overline{1 \mathrm{~s}}$
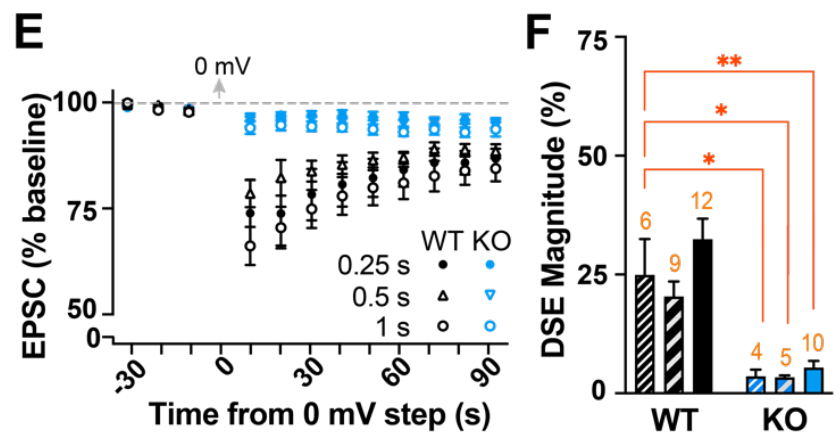

Figure 1 Depolarization-Induced Suppression of Excitation (DSE) reduces climbing fiberEPSC amplitude in WT but not $\alpha 2 \delta-2$ KO Purkinje cells.

A) Experimental schematic. Purkinje cell (PC) is held at $-70 \mathrm{mV}$ while the climbing fiber axon (red) is stimulated at $0.2 \mathrm{~Hz}$ (arrows). A depolarization step to $0 \mathrm{mV}$ is delivered to the PC between EPSC recordings.

B) Overlay of peak-scaled EPSC traces during baseline (grey) and $5 \mathrm{~s}$ post-depolarization (dotted line) from WT (black) and KO (blue) PCs; 250 ms step duration.

C) EPSC amplitudes from experiment in B. Each point averaged two consecutive EPSCs.

D) Overlay of peak-scaled EPSCs during baseline (grey) and $5 \mathrm{~s}$ after $250 \mathrm{~ms}$ (dotted line), 500 $\mathrm{ms}$ (dashed line), and $1 \mathrm{~s}$ (solid line) depolarization from WT (black) and KO (blue) PCs.

E) DSE timecourse from WT (black) and KO (blue) experiments using $250 \mathrm{~ms}$ (filled circle), 500 $\mathrm{ms}$ (triangle) and $1 \mathrm{~s}$ (open circle) depolarizing step lengths.

F) EPSC depression normalized to baseline in WT (black) and KO (blue) after $250 \mathrm{~ms}$ (fine stripe), $500 \mathrm{~ms}$ (wide stripe) and $1 \mathrm{~s}$ (solid) depolarization steps. $\mathrm{n}=$ cells (orange number over bars); each experiment from > 3 mice. One-way ANOVA comparison to average WT 250 ms response, Dunnett's correction for multiple comparisons; ${ }^{*} p<0.05,{ }^{* *} p<0.01,{ }^{* * *} p<$ 0.001 . 

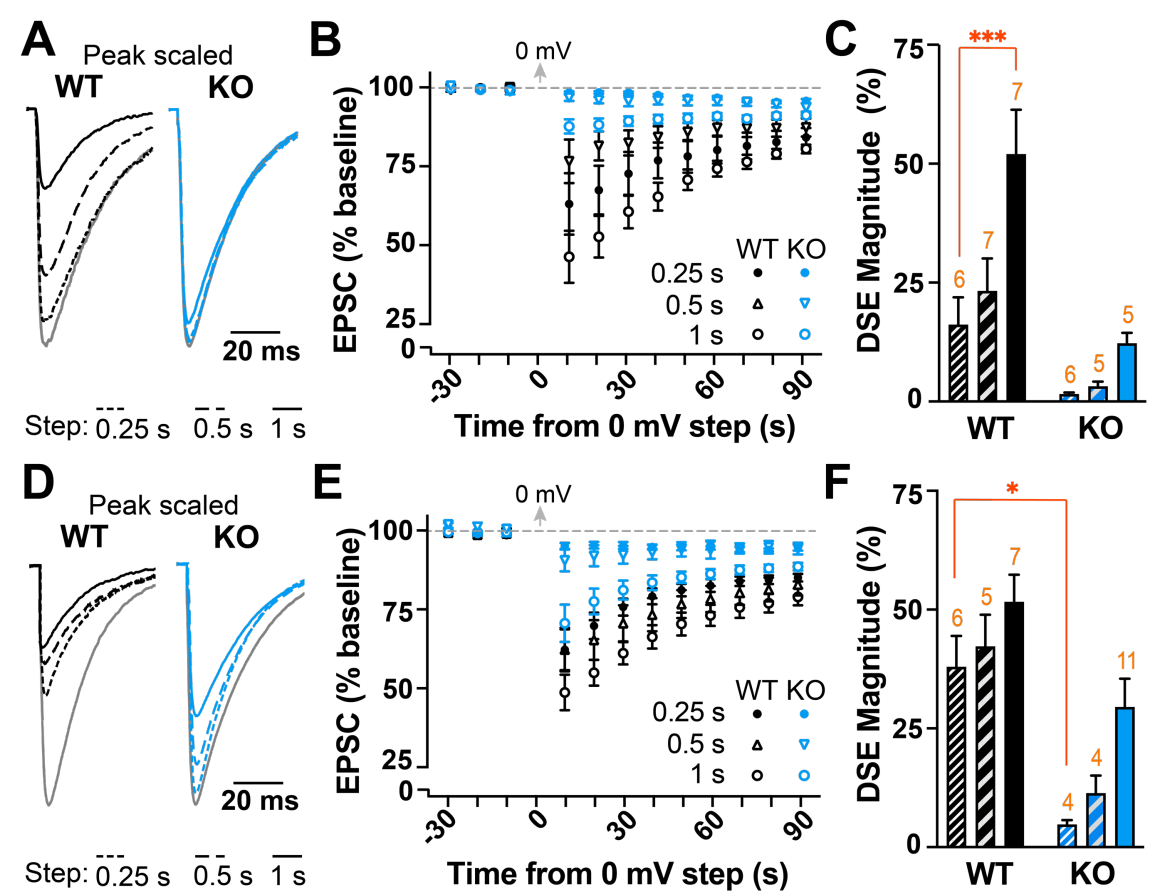

Figure 2 Reduced intracellular [EGTA] reveals DSE in the $\alpha 2 \delta-2 \mathrm{KO}$.

A, D) Overlay of peak-scaled EPSCs during baseline (grey) and $5 \mathrm{~s}$ after $250 \mathrm{~ms}$ (dotted line), $500 \mathrm{~ms}$ (dashed line), and $1 \mathrm{~s}$ (solid line) depolarization using intracellular solution containing 2 $\mathrm{mM}$ (A) or $0.2 \mathrm{mM}$ (D) EGTA from WT (black) and KO (blue) PCs.

B, E) DSE timecourse from WT (black) and KO (blue) experiments using a $2 \mathrm{mM}$ (B) or 0.2 $\mathrm{mM}$ (E) EGTA and depolarization steps of $250 \mathrm{~ms}$ (filled circle), $500 \mathrm{~ms}$ (triangle) and $1 \mathrm{~s}$ (open circle).

C, F) EPSC depression normalized to baseline in WT (black) and KO (blue) after $250 \mathrm{~ms}$ (fine stripe), $500 \mathrm{~ms}$ (wide stripe) and $1 \mathrm{~s}$ (solid) depolarization steps using a $2 \mathrm{mM}$ (C) or $0.2 \mathrm{mM}$ (F) EGTA. $n=$ cells (orange number over bars); each experiment from $>3$ mice. One-way ANOVA comparison to average WT $250 \mathrm{~ms}$ response, Dunnett's correction for multiple comparisons; ${ }^{*} p<0.05,{ }^{* *} p<0.01,{ }^{* * *} p<0.001$. 

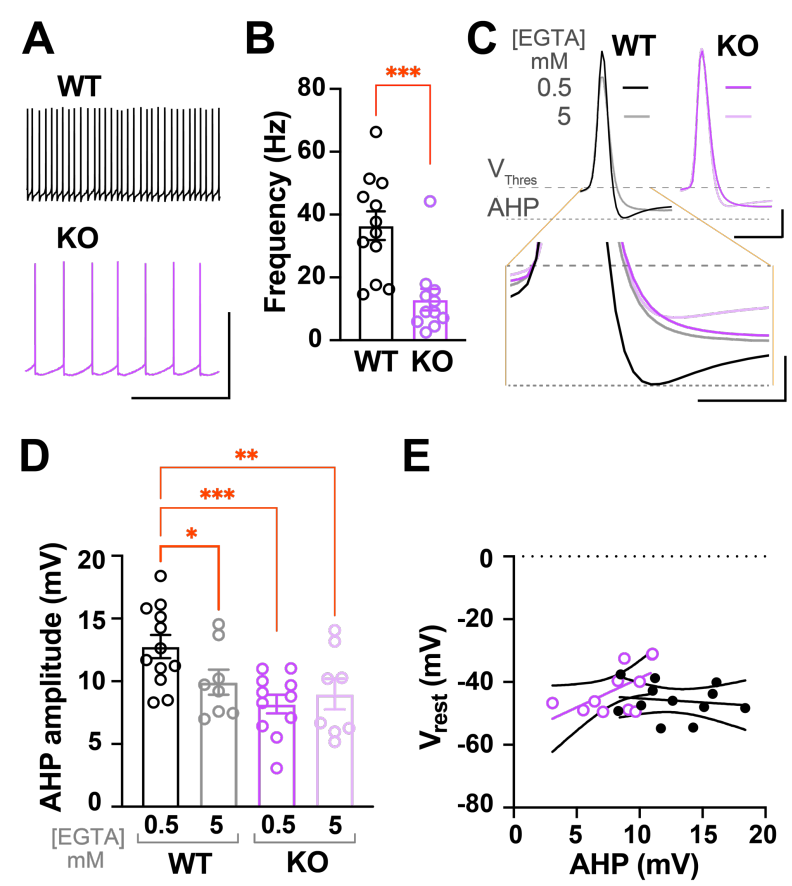

E
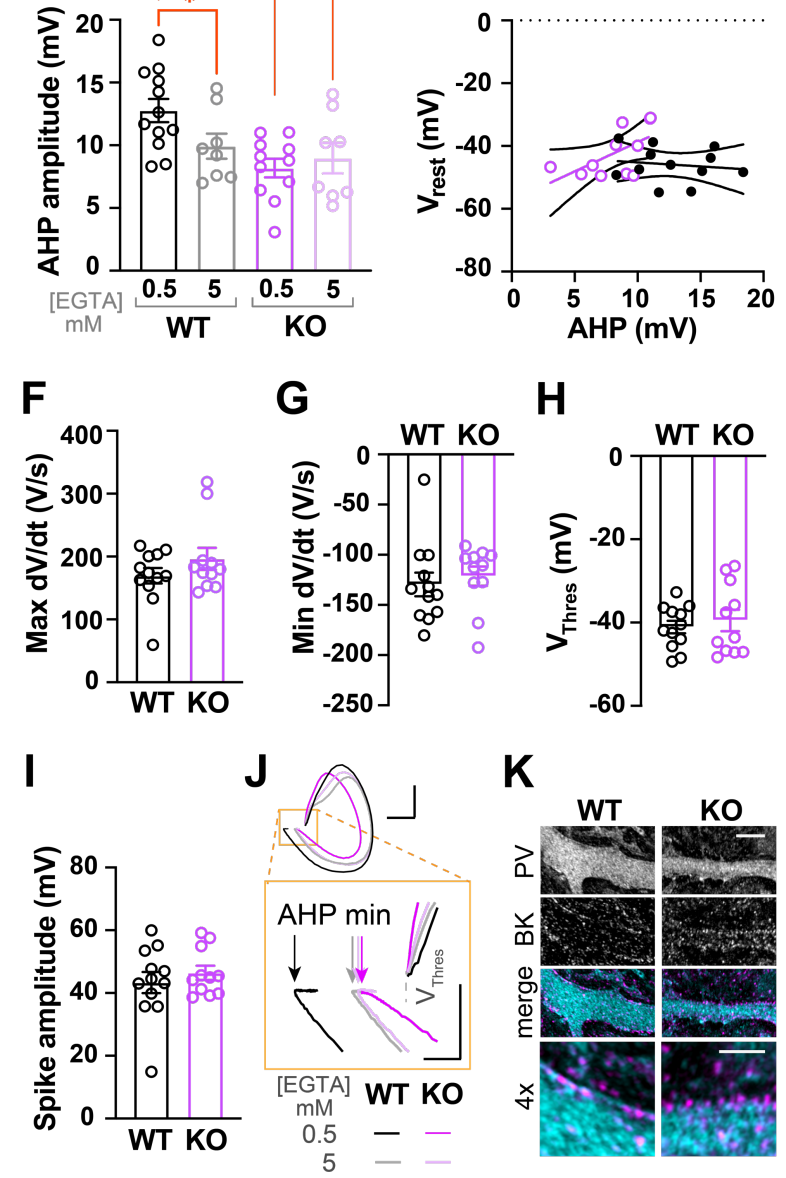

Figure 3 Spontaneous firing frequency, afterhyperpolarization (AHP) amplitude and $\mathrm{Ca}^{2+}$ coupling are reduced in the $\alpha 2 \delta-2 \mathrm{KO}$.

A) Spontaneous spikes in WT (black) and KO (magenta) PCs; scale: $50 \mathrm{mV}, 0.5 \mathrm{~s}$.

B) Spontaneous firing frequency in PCs. Student's unpaired t-test, $p<0.0001$.

C) Averaged spontaneous spikes from WT and KO PCs during tonic firing (0.5 mM EGTA, black/magenta; $5 \mathrm{mM}$ EGTA grey/light purple); scale: $10 \mathrm{mV}, 2 \mathrm{~ms}$. Grey dotted lines indicate $V_{\text {thres }}$ and minimum voltage during AHP. Below, enlarged overlay demonstrating differences in AHP amplitude; scale: $5 \mathrm{mV}, 1 \mathrm{~ms}$. 
D) AHP amplitudes recorded using $0.5 \mathrm{mM}$ EGTA or $5 \mathrm{mM}$ EGTA intracellular solution. One-way ANOVA comparison to average WT 0.5 mM EGTA response, uncorrected Fischer's LSD; * $p<$ $0.05,{ }^{* *} p<0.01,{ }^{* * *} p<0.001$.

E) Correlation of AHP amplitude vs. resting membrane potential $\left(\mathrm{V}_{\mathrm{m}}\right)$ in WT (black) and KO (magenta) PCs using 0.5 mM EGTA. Linear regression and 95\% confidence interval; WT $R^{2}=$ $<0.002 ; \mathrm{KO} \mathrm{R}^{2}=0.393$.

F-I) Spike waveform parameters in WT and KO PCs. No differences in maximum dV/dt (F), minimum dV/dt $(\mathbf{G})$, spike threshold $\left(\mathrm{V}_{\text {thres }}\right)(\mathbf{H})$ or spike height $(\mathbf{I})$; unpaired Student's t-tests.

J) Phase plane plots of spontaneous spikes in WT and KO PCs (0.5 mM EGTA, black/magenta; 5 mM EGTA grey/light purple). Traces aligned by spike threshold $\left(V_{\text {thresh }}\right)$ for comparison; scale: $100 \mathrm{mV} / \mathrm{ms}, 20 \mathrm{mV}$. Below, enlarged inset to illustrate differences in AHP minimum amplitude (arrows; $0.5 \mathrm{mM}$ EGTA, black/magenta; $5 \mathrm{mM}$ EGTA, grey/light purple); scale: $100 \mathrm{mV} / \mathrm{ms}, 5 \mathrm{mV}$.

K) Immunohistochemistry of WT and KO cerebellar slices stained for parvalbumin (PV; cyan) and the BK channel (magenta); scale: $5 \mu \mathrm{m}$. Below, merged higher power image; scale: $2 \mu \mathrm{m}$. 